\title{
Some symmetric varieties of groups
}

\section{Narain Gupta and Frank Levin}

Let $\underline{\underline{v}}(n, \sigma, d)$ denote the variety of all groups defined by the left-normed commutator identity $\left[x_{1}, \ldots, x_{n}\right]=\left[x_{1 \sigma}, \ldots, x_{n \sigma}\right]^{d}$, where $\sigma$ is a non-identity permutation of $\{1, \ldots, n\}$, and $d$ is an integer, possibly negative. It is shown that $\underline{\underline{V}}(n, \sigma, d)$ is nilpotent-by-rilpotent if $\sigma \neq(1,2)$, abelian by nilpotent if $n>2, n \sigma \neq n$, and nilpotent of class at most $n+1$ if $\{1,2\} \neq\{1 \sigma, 2 \sigma\}$. This improves on a result of E.B. Kikodze that $\underline{\underline{V}}(n, \sigma, 1)$ is locally soluble and if $\{1,2\} \neq\{1 \sigma, 2 \sigma\}$ is locally nilpotent.

\section{Introduction}

In the present note we consider groups satisfying a left-normed commutator identity

$$
\left[x_{1}, \ldots, x_{n}\right]=\left[x_{10}, \ldots, x_{n \sigma}\right]^{d},
$$

where $\sigma$ is a non-identity permutation of $\{1, \ldots, n\}$ and $d$ is an integer. E.B. Kikodze [2] has shown that any finitely generated group satisfying (1) with $d=1$ is soluble and is nilpotent if $\{1,2\} \neq\{1 \sigma, 2 \sigma\}$. The following theorems extend these results to arbitrary $d$ without the assumption of finite generation.

THEOREM 1. Let $G$ be a group satisfying an identity (1) for some $\sigma$ such that $\{1,2\} \neq\{1 \sigma, 2 \sigma\}$. Then $G$ is nilpotent of class at most $n+1$.

Received 23 April 1970. The authors gratefully acknowledge support from the National Research Council and the National Science Foundation, respectively. 
THEOREM 2. Let $G$ satisfy (1) for some $n \geq 3$ and $\sigma \neq(12)$. Then for some integers $m, k, G$ satisfies $\left[\Gamma_{m}(G), \Gamma_{2}(G), x_{1}, \ldots, x_{k}\right]=1$, where $\Gamma_{m}(G)$ denotes the $m$-th term of the lower central series of $G$. In particular, $G$ is nilpotent-by-nilpotent.

Note. Theorem 2 extends to $\sigma=(12)$ for $d=1$ by a result of I.D. Macdonald [5].

THEOREM 3. Let $G$ satisfy (1) for some $n \geq 3$ and no $\neq n$. Then for some $m, G$ satisfies $\left[\Gamma_{m}(G), \Gamma_{2}(G)\right]=1$ and, hence, is abelian-by-nilpotent.

The proofs of these results are given in Section 4. Before closing this introduction we record some of the more pertinent known results concerning groups $G$ satisfying (1) with $d=1$.
(A) $\left[x_{1}, x_{2}\right]=\left[x_{2}, x_{1}\right]$ implies $\left[\Gamma_{3}(G), \Gamma_{2}(G)\right]=1$ (Macdonald [5]).
(B) $\left[x_{1}, x_{2}, x_{1}\right]=1$ implies nilpotence class $\leq 3$ (Levi [3]).
(C) For $m \geq 3, \sigma=(n-1, n)$, (I) implies $\left[\Gamma_{n-2}(G), \Gamma_{2}(G)\right]=1$ (Levin [4]).
(D) For $n \geq 4, \sigma=(123 \ldots n)$, (1) implies nilpotence class $\leq n-1$ (Macdonald [5] and Meir-Wunderli (cf. [5])).

\section{Notation}

For unexplained notation we refer the reader to Hanna Neumann [6]. In particular, for elements $a, b, c, \ldots$ in a group $A$ we define $[a, b]=a^{-1} b^{-1} a b,[a, b, c]=[[a, b], c], a^{b}=b^{-1} a b$. If $H, K$ are subgroups of $A$, then $[H, K]$ denotes the subgroup of $A$ generated by all $[h, k], h \in H, k \in K$. Further, we define $[H, O K]=H$ and for $t \geq 1,[H, t K]=[[H,(t-1) K], K]$. Finally, $\Gamma_{m}(A)\left(\zeta_{m}(A)\right)$ denotes the $m$-th term of the lower (upper) central series of $A$. 


\section{Two lemmas}

LEMMA 3.1. Let $u, v$ be fixed elements of a group $G$ such that for some $r \geq 1$ and all $x_{1}, \ldots, x_{r} \in G$

$$
\left[u, x_{1}, \ldots, x_{r}, v\right]=1 \text {. }
$$

Then $[U, s G, V]=\{I\}$ for any $s \geq r$, where $U$ and $V$ are the normal closures of $u$ and $v$, respectively.

Proof. Replacing $x_{r}$ by $x_{p} g^{-1}$ in (2) and expanding (using the identity $\left.[a, b c]=[a, c][a, b]^{c}\right)$ gives $\left[\left[u, x_{1}, \ldots, x_{p}\right]^{g^{-1}}, v\right]=1$, which after conjugation with $g$, gives $\left\lfloor u, x_{1}, \ldots, x_{p}, v^{g}\right\rfloor=1$. If we now replace $g$ by $g h^{-1}, x_{i}$ by $x_{i}^{h^{-1}}$ for $i=1, \ldots, r$, and conjugate the resulting expression by $h$, we obtain $\left[u^{h}, x_{1}, \ldots, x_{r}, v^{g}\right]=1$. Hence, $[U, r G, V]=\{1\}$. In particular $\left[\left[u^{h}, x_{r+1}, \ldots, x_{s}\right], x_{1}, \ldots, x_{r}, v^{g}\right]=1$ for any $s>r$, so that $[U, s G, V]=\{I\}$, as required.

LEMMA 3.2. Let $u, v$ be fixed elements of a group $G$ which satisfies an identity (1) with $1 \sigma=1,2 \sigma=2$ and $n \sigma=n-i \neq n$. If $G$ satisfies

$$
\left[u, x_{2}, \ldots, x_{r}, v, x_{p+2}, \ldots, x_{k}\right]=1
$$

for some $k \geq r+1, r \geq 2$, and all $x_{i} \in G$, then for some integer $t$,

$$
[U, t G, V]=\{1\} \text {. }
$$

Proof. By Lemma 3.1, $G$ satisfies

$$
\left[u_{1}, x_{2}, \ldots, x_{n-i-1}, v, x_{n-i+1}, \ldots, x_{m}\right]=1 \text {, }
$$

with $u_{1}=u$ if $r \leq n-i-1$ and sufficiently large $m$. Otherwise, $G$ satisfies (4) with $u_{1}=\left[u, y_{2}, \ldots, y_{r-(n-i-1)}\right]$. Choose $m$ of the form $n+p(n-i)$. By (4), $G$ satisfies, in particular, 
$\left[\left[u_{1}, x_{2}, \ldots, x_{n-i-1}, v, \ldots, x_{n}\right]^{d}, \ldots, x_{m}\right]=1$ so that, by (1), $G$ satisfies $\left[u_{1}, x_{2}, \ldots, x_{n-1}, v, \ldots, x_{m}\right]=1$. Iterating this process $p$ times yields $\left[u_{1}, \ldots, x_{m}, v\right]=1$ which, by Lemma 3.1 , proves the lemma.

Finally, we remark that (1) implies $\left[x_{1}, \ldots, x_{n}\right]=\left[x_{1 \rho}, \ldots, x_{n \rho}\right]^{d^{N-1}}$, where $\rho=\sigma^{-1}$ and $N$ is the order of $\sigma$.

\section{Proofs of the theorems}

Proof of Theorem 1: Case 1. $\{1 \sigma, 2 \sigma\}=\{i, j\}$ and $\{i, j\} \cap\{1,2\} \neq \emptyset$. In (1) replacing $x_{i}$ by $x_{1}$ or $x_{2}$ shows that $G$ satisfies the law $\left[x_{1}, x_{2}, \ldots, x_{i}, x_{1}, x_{i+1}, \ldots, x_{n}\right]=1$ or $\left[x_{2}, x_{1}, \ldots, x_{i-1}, x_{1}, x_{i+1}, \ldots, x_{n}\right]=1$. Thus, if $i=3, G$ is nilpotent of class $\leq n$ by the Levi result [3], while if $i>3, G$ is nilpotent of class $\leq n-1$ by a result of Heineken and Macdonald ( $c f$. 34.33 of [6]).

Case 2. $\{1 \sigma, 2 \sigma\}=\{i, j\},\{i, j\} \cap\{1,2\}=\emptyset, i<j$. Replacing $x_{j}$ by $x_{i}$ in (l) shows that $G$ satisfies the law

$$
\left[x_{1}, x_{2}, \ldots, x_{i}, \ldots, x_{j-1}, x_{i}, x_{j+1}, \ldots, x_{n}\right]=1 \text {. }
$$

If $j=i+1$, then, replacing $\left[x_{1}, x_{2}\right]$ by $\left[x_{1}, x_{2}, y\right]$, shows that $G$ satisfies

$$
\left[x_{1}, x_{2}, \ldots, x_{i}, x_{i+1}, x_{i+3}, \ldots, x_{n}, x_{n+1}\right]=1
$$

so that

(6) $\left[\left[x_{1}, x_{2}, \ldots, x_{i}, x_{i+1}, x_{i+1}, x_{i+3}, \ldots, x_{n}\right]^{d^{N-1}}, x_{n+1}\right]=1$, where $N$ is the order of $\sigma$. By the remark at the end of Section 3 we may apply $\sigma$ to the indexes of the first $n x^{\prime} s$ in (6) to obtain an identity of the form

$$
\left[x_{1}, x_{i+1}, \ldots, x_{i+1}, \ldots, x_{n}, x_{n+1}\right]=1
$$


which implies, as in Case 1 , that $G$ is nilpotent of class $\leq n+1$. Finally, if $j>i+1$ we may apply Lema 3.1 to (5) in order to insert one extra component between the two $x_{i}$ 's to obtain an identity

$$
\left[x_{1}, x_{2}, \ldots, x_{i}, \ldots, x_{j}, x_{i}, x_{j+1}, \ldots, x_{n}, x_{n+1}\right]=1 \text {, }
$$

so that, as in (6),

$$
\left[\left[x_{1}, x_{2}, \ldots, x_{i}, \ldots, x_{j}, x_{i}, x_{j+1}, \ldots, x_{n}\right]^{d^{N-1}}, x_{n+1}\right]=1 \text {, }
$$

which, as above, implies that $G$ is nilpotent of class $\leq n+1$. This completes the proof of Theorem 1 .

Proof of Theorem 2: Case 1. $d=1,1 \sigma=1,2 \sigma=2$. Here we may assume that $G$ satisfies the law

$$
\left[u, x_{1}, \ldots, x_{n}\right]=\left[u, x_{1 \sigma}, \ldots, x_{n \sigma}\right] \text {, }
$$

where $u=\left[y_{1}, \ldots, y_{p}\right](r \geq 2)$ and $1 \sigma=i \neq 1$. Replacing $x_{i}$ by $u$ in (7) yields the law

$$
\left[u, x_{1}, \ldots, x_{i-1}, u, x_{i+1}, \ldots, x_{n}\right]=1 \text {, }
$$

which, by Lemma 3.1 , implies that $[U,(i-1) G, U,(n-i) G]=\{1\}$, where $U$ is the normal closure of $u$ in $G$.

In particular, $G_{1}=G / \zeta_{n-i}(G)$ satisfies the identity

$$
\left[u^{g}, x_{1}, \ldots, x_{p}, u^{h}\right]=1
$$

where $p \geq i-1$. In (7) we may replace $u$ by $v=\left[y_{1}, \ldots, y_{p+i-1}\right]$ to obtain

$$
\left[v, x_{1}, \ldots, x_{n}\right]=\left[v, x_{1 \sigma}, \ldots, x_{n \sigma}\right] .
$$

If we now replace $x_{i}$ by $z_{i} x_{i}$ in (10) and expand using (9), we find that $G$ satisfies

(II) $\left[v, x_{1}, \ldots, x_{i-1}, z_{i}, x_{i}, x_{i+1}, \ldots, x_{n}\right]$

$$
\begin{aligned}
& =\left[v, z_{i}, x_{i}, x_{2 \sigma}, \ldots, x_{n \sigma}\right] \\
& =\left[v, z_{i}, x_{1}, \ldots, x_{n}\right],
\end{aligned}
$$


by applying (10) to the last $n$ entries of the right hand commutator. Thus, $G_{2}=G_{1} / \zeta_{n-i+1}\left(G_{1}\right)$ satisfies the law

$$
\left[v, x_{1}, \ldots, x_{i-1}, x_{i}\right]=\left[v, x_{i}, x_{1}, \ldots, x_{i-1}\right] \text {, }
$$

by Lema 1 of [2] (which states that $G$ satisfies

$\left[w_{1}, x_{1}, \ldots, x_{k}\right]=\left[w_{2}, x_{1}, \ldots, x_{k}\right]$ if and onily if $G / \zeta_{k}(G)$ satisfies $w_{1}=w_{2}$ ). In particular, commuting both sides of (12) by $x_{i+1}$ gives

$$
\left[v, x_{1}, \ldots, x_{i}, x_{i+1}\right]=\left[v, x_{i}, x_{1}, \ldots, x_{i-1}, x_{i+1}\right] .
$$

Two applications of (12) to the last $i$ entries of the left side of (13) and one application of (12) to the last $i$ entries of the right side of (13) now give the law

(14) $\left[v, x_{1}, x_{i}, x_{i+1}, x_{2}, \ldots, x_{i-1}\right]=$

$$
\left[v, x_{i}, x_{i+1}, x_{1}, x_{2}, \ldots, x_{i-1}\right] \text {, }
$$

so that again, by Lemma 1 of [2], $G_{3}=G_{2} / \zeta_{i-2}\left(G_{2}\right)$ satisfies the law

$$
\left[v, x_{1}, x_{2}, x_{3}\right]=\left[v, x_{2}, x_{3}, x_{1}\right] \text {. }
$$

Replacing $x_{3}$ by $x_{3} x_{4}$ in (15) and expanding using (9) gives

$$
\begin{aligned}
& {\left[v, x_{1}, x_{2}, x_{3}, x_{4}\right]=\left[v, x_{2}, x_{3}, x_{4}, x_{1}\right] } \\
&=\left[\left[v, x_{2}\right], x_{4}, x_{1}, x_{3}\right], \text { by (15), } \\
&=\left[v, x_{2}, x_{1}, x_{3}, x_{4}\right], \text { by (15). }
\end{aligned}
$$

Thus, $G_{4}=G_{3} / \zeta_{2}\left(G_{3}\right)$ satisfies the law

$$
\left[v, x_{1}, x_{2}\right]=\left[v, x_{2}, x_{1}\right],
$$

which by a result of Levin [4] implies that $\left[\Gamma_{p+i-1}\left(G_{4}\right), \Gamma_{2}\left(G_{4}\right)\right]=\{1\}$. Since $G_{4}=G / \zeta_{k}(G)$ where $k=2+(i-2)+(n-i+1)+(n-i)=2 n-i+1$, it follows that $\left[\Gamma_{p+i-1}(G), \Gamma_{2}(G)\right] \leq \zeta_{k}(G)$, or, equivalently, that $\left[\Gamma_{r+i-1}(G), \Gamma_{2}(G), k G\right]=\{1\}$.

Case 2. $1 \sigma=1,2 \sigma=2, d$ arbitrary. As in Case 1 we may assume that $G$ satisfies the law

$$
\left[u, x_{1}, \ldots, x_{n}\right]=\left[u, x_{1 \sigma}, \ldots, x_{n \sigma}\right]^{d} \text {, }
$$


where $u=\left[y_{1}, \ldots, y_{p}\right], r \geq 2, I \sigma=i \neq 1$, and as in Case $I$ this implies that $G_{1}=G / \zeta_{n-i}(G)$ satisfies $(9)$ and

$$
\left[v, x_{1}, \ldots, x_{n}\right]=\left[v, x_{1 \sigma}, \ldots, x_{n \sigma}\right]^{d} \text {, }
$$

where $v=\left[y_{1}, \ldots, y_{p+i-1}\right]$. Again replecing $x_{i}$ by $z_{i} x_{i}$ in (17) and expanding, using (9), yields

$$
\begin{aligned}
{\left[v, x_{1}, \ldots, x_{i-1}, z_{i}, x_{i}, x_{i+1}, \ldots, x_{n}\right] } & \\
& =\left[v, z_{i}, x_{i}, x_{2 \sigma}, \ldots, x_{(n-1) \sigma}, x_{n \sigma}\right]^{d} \\
& =\left[v, z_{i}, x_{1}, \ldots, x_{n}\right],
\end{aligned}
$$

as above. From this point on the proof is identical with that in Case 1.

Case 3. $\quad 1 \sigma=2,2 \sigma=1, \sigma \neq(12)$. As above, we may assume that $G$ satisfies the law

$$
\left[u, x_{1}, \ldots, x_{n}\right]=\left[v, x_{1 \sigma}, \ldots, x_{n \sigma}\right]^{d},
$$

where $u=\left[y_{1}, y_{2}, \ldots, y_{p}\right], v=\left[y_{2}, y_{1}, y_{3}, \ldots, y_{p}\right], r \geq 2$, and $1 \neq 1 \sigma=i$. In (18) replacing $x_{i}$ by $v$ gives $\left[u^{g}, x_{1}, \ldots, x_{i-1}, v, x_{i+1}, \ldots, x_{n}\right]=1$ so that $G_{1}=G / \zeta_{n-i}{ }^{(G)}$ satisfies the law

$$
\left[u^{g}, x_{1}, \ldots, x_{n}, v^{n}\right]=1
$$

so that, in particular,

$$
\left[\left[u^{g}, x_{1}, \ldots, x_{n}\right]^{d}, v^{h}\right]=1 .
$$

Thus, by (18), $G_{1}$ satisfies

$$
\left[v^{g}, x_{1}, \ldots, x_{n}, v^{n}\right]=1
$$

Further, in (18) replacing $y_{2}$ by $y_{2}^{*}=\left[y_{2,1}, \ldots, y_{2, n-r}\right]$ shows that $G_{1}$ satisfies the law 


$$
\left[u^{*}, x_{1}, \ldots, x_{n}\right]=\left[v^{*}, x_{1 \sigma}, \ldots, x_{n \sigma}\right]^{d}
$$

where $u^{*}=\left[y_{1}, y_{2}^{*}, y_{3}, \ldots, y_{p}\right], v^{*}=\left[y_{2}^{*}, y_{1}, y_{3}, \ldots, y_{r}\right]$. Let $k$ be the largest integer in $\{1, \ldots, n\}$ such that $k \sigma \neq k$. Then in (21) replace $x_{k \sigma}$ by $x_{k \sigma} x_{n+1}$ and expand both sides (using (20)) to obtain

$$
\begin{aligned}
{\left[u^{*}, x_{1}, \ldots, x_{k}, x_{n+1}, \ldots, x_{n}\right] } & =\left[v^{*}, \ldots, x_{k \sigma}, x_{n+1}, \ldots, x_{n \sigma}\right]^{d} \\
& =\left[\left[v^{*}, \ldots, x_{k \sigma}, x_{n+1}, \ldots, x_{(n-1) \sigma}\right]^{d}, x_{n \sigma}\right],
\end{aligned}
$$

which, after an application of (21) to the latter commutator, yields an identity of the type considered in the previous cases. This completes the proof of Theorem 2 .

Proof of Theorem 3. Theorem 3 follows immediately from Theorem 2 and Lenma 3.2 .

\section{Generalization}

Let $w\left(x_{1}, \ldots, x_{n}\right)$ be a word which reduces to the identity if $x_{i_{1}}=\ldots=x_{i_{k}}=x_{1}$ for some sequence $3 \leq i_{1}<\ldots<i_{k} \leq n$ satisfying $i_{j}<i_{j+1}-1$. If $G$ is a group satisfying the law

$$
\left[x_{1}, \ldots, x_{n}\right]=\omega\left(x_{1}, \ldots, x_{n}\right) \text {, }
$$

then $G$ satisfies the law $\left[x_{1}, \ldots, x_{n}\right]=1$ with $x_{i_{1}}=\ldots=x_{i_{k}}=x_{1}$, and by Lemma 2 of Kikodze [2] it follows that, in particular, $G$ satisfies the law $\left[\left[x_{1}, \ldots, x_{d}, y\right], \ldots,\left[x_{1}, \ldots, x_{d}, y_{m}\right]\right]=1$ for some integers $m, d \leq n$. By Lemma 7 of N.D. Gupta [1] it then follows that $G$ is locally nilpotent-by-nilpotent. Thus, every group of the variety defined by (22) is locally nilpotent-by-nilpotent.

\section{References}

[1] N.D. Gupta, "Certain locally metanilpotent varieties of groups", Arch. Math. 20 (1969), 481-484. 
[2] E.B. Kikodze, "Some identities in groups" (English), Math. USSR Izvestija 1 (1967), 253-258.

[3] F.W. Levi, "Groups in which the commutator relation satisfies certain algebraic conditions", J. Indian Math. Soc. (N.S.) 6 (1942), $87-97$.

[4] Frank Levin, "On some varieties of soluble groups. II", Math. 2. 103 (1968), 162-172.

[5] I.D. Macdonald, "On certain varieties of groups", Math. 2. 76 (1961), $270-282$.

[6] Hanna Neumann, Varieties of groups (Ergebnisse der Mathematik und ihrer Grenzgebiete, Band 37, Springer-Verlag, Berlin, Heidelberg, New York, 1967).

University of Manitoba,

Winnipeg, Canada,

and

Rutgers, The State University,

New Brunswick, New Jersey,

USA. 